\title{
American Joint Committee on Cancer's Staging System for Breast Cancer, Eighth Edition: Summary for Clinicians
}

\author{
(1) Haoling Zhu, (1) Başak E. Doğan \\ Department of Radiology UT Southwestern Medical Center, Dallas, Texas
}

\begin{abstract}
Breast cancer is commonly staged using the American Joint Committee on Cancer (AJCC) staging system. The $7^{\text {th }}$ edition of the AJCC Staging Manual, was a purely anatomic staging method, which uses primary tumor size $(\mathrm{T})$, nodal involvement $(\mathrm{N})$, and metastasis $(\mathrm{M})$ based on clinical and pathological evaluations. Advancements in tumor biology and prognostic biological markers, such as estrogen receptor (ER)/progesterone receptor (PR), HER2/neu, and $\mathrm{Ki}-67$, have allowed clinicians to understand why similarly staged patients had significantly different outcomes. The most recent update to the staging system integrates molecular markers with disease extent for more optimal estimation of prognosis. This change improves the prognosis of breast cancer patients and better informs physicians in the planning of treatments. This review summarizes the changes in the AJCC Staging Manual, $8^{\text {th }}$ edition and their impact on practicing radiologists in breast cancer management.
\end{abstract}

Keywords: AJCC, biomarkers, Breast cancer, Breast imaging, cancer staging

Cite this article as: Zhu H, Doğan BE. American Joint Committee on Cancer's Staging System for Breast Cancer, Eighth Edition: Summary for Clinicians. Eur J Breast Health 2021; 17(3): 234-238

\section{Key Points}

- Until the implementation of the $8^{\text {th }}$ edition of the AJCC Staging Manual, a purely anatomic staging method, which uses primary tumor (T) size, nodal $(\mathrm{N})$ involvement, and metastasis $(\mathrm{M})$ based on clinical and pathological evaluations, was employed.

- Advancements in tumor biology and prognostic biological markers, such as estrogen receptor (ER)/progesterone receptor (PR), HER2/neu, and Ki-67, have allowed clinicians to understand why similarly staged patients had significantly different outcomes.

- The most recent update to the staging system integrates molecular markers with disease extent for more optimal estimation of prognosis.

\section{Introduction}

Breast cancer is the most diagnosed cancer among women in the United States (US). It is also the leading cause of cancer-related deaths following lung cancer. In 2020, 276,480 new cases of breast cancer were projected to be diagnosed among American women along with 48,530 new cases of non-invasive breast cancer. One out of eight American women is projected to develop invasive breast cancer at some point in her life (1). In 1959, the American Joint Committee for Cancer Staging and End-Results Reporting, now the American Joint Committee on Cancer (AJCC), standardized the tumor, node, and metastasis (TNM) cancer staging system. The first edition of the AJCC Staging Manual, published in 1977, allowed clinicians to standardize treatment and evaluate treatment results between different institutions $(2,3)$. Since then, the manual has been periodically updated to reflect clinical and technological advancements in the field.

Until the implementation of the 8th edition of the AJCC Staging Manual in 2018, a purely anatomic staging method, which uses primary tumor $(\mathrm{T})$ size, nodal $(\mathrm{N})$ involvement, and metastasis $(\mathrm{M})$ based on clinical and pathological evaluations, was employed. Advancements in tumor biology and prognostic biological markers [estrogen receptor (ER) and progesterone receptor (PR), HER2/neu, and Ki-67] have allowed clinicians to understand why similarly staged patients had significantly different outcomes. The most recent update to the staging system integrates anatomic staging with prognostic staging, which uses tumor grade, hormone receptors and oncogene expression, and multigene testing (4). Incorporating the prognostic stage into the breast cancer staging system has allowed physicians to individualize the patient prognosis, leading to a more optimal estimation of prognosis. 
This article highlights key changes from the 7 th edition to the 8th edition of the AJCC breast cancer staging system, with multimodality imaging demonstration of its application. We will review the anatomic TNM staging categories: clinical staging, pathologic staging, post-therapy or post-neoadjuvant therapy staging, and restaging. Furthermore, we will summarize the prognostic staging (both clinical and pathological), the implementation of gene assays, and how they are integrated in different scenarios. Other changes in the AJCC Staging Manual, $8^{\text {th }}$ Edition, will also be discussed, including re-categorization of lobular carcinoma in situ (LCIS) and the clinical N stage based on physical examination and imaging studies (4).

\section{Anatomic staging}

Anatomic TNM staging is further categorized into: clinical staging, which relies on physical examination, imaging, and biopsy of the affected areas; pathologic staging, which is determined after a patient has had surgery to remove the primary tumor and regional lymph nodes; post-neoadjuvant therapy staging, which determines how much cancer remains after a patient completes preoperative systemic or radiation therapy, and may incorporate both clinical and pathologic staging; and restaging, which is performed if a cancer recurs after treatment and determines the extent of disease recurrence. Since this review is primarily for radiologists, we will spend most of this section discussing clinical anatomic staging. Importantly, imaging findings are considered relevant to staging if obtained within 4 months of diagnosis or completion of surgery, whichever confers a longer time period, provided the disease has not worsened.

\section{Clinical anatomic staging}

Clinical staging of the primary $\mathrm{T}$ begins with the measurement of the tumor size based on physical examination and imaging (4). The staging categories range from Tis to T4 and are the same for both clinical and pathological staging of the primary tumor, where the prefixes " $c$ " and "p" indicate clinical stage and pathologic stage, respectively. Tis refers to ductal carcinoma in situ with no invasive cancer. T1-T4 refers to the tumor size, ranging from $2 \mathrm{~cm}$ to $>5 \mathrm{~cm}$, and the involvement of chest wall and/or skin (ulceration or macroscopic nodules), respectively. The T4 category is further subdivided into T4a-T4d, where T4a indicates chest wall involvement; T4b skin indicates involvement through ulceration, ipsilateral macroscopic satellite nodules, and/or skin edema (e.g., peau d'orange), which does not meet the criteria for inflammatory carcinoma; T4c is indicated when both T4a and T4b are present; and T4d inflammatory carcinoma. Lastly, LCIS is no longer staged via TNM in the Tis category, as it is now viewed as benign; however, it carries a risk of future malignancy.

Similar to primary tumor staging, clinical staging of regional axillary lymph nodes $(\mathrm{N})$ should begin with the prefix "c." The staging categories range from $\mathrm{N} 0$ to $\mathrm{N} 3$, where $\mathrm{N} 0$ indicates no regional lymph node metastases as revealed by imaging or clinical examination; $\mathrm{N} 1$ indicates metastases to movable ipsilateral level I-II [where level I nodes are lateral to the lateral border of the pectoralis minor muscle and level II nodes are between the medial and lateral borders of the pectoralis minor and also include the interpectoral (Rotter's) lymph nodes] axillary lymph nodes; N2 indicates metastases to ipsilateral level I-II axillary nodes that are clinically fixed or matted, or metastases to ipsilateral internal mammary nodes without axillary lymph node involvement ( $\mathrm{N} 2 \mathrm{a}$ and $\mathrm{N} 2 \mathrm{~b}$, respectively); $\mathrm{N} 3$ indicates metastases to level III (ipsilateral infraclavicular) lymph nodes, ipsilateral internal mammary lymph nodes with level I-II axillary node metastases, or metastases to ipsilateral supraclavicular lymph nodes (N3a, N3b, and $\mathrm{N} 3 \mathrm{c}$, respectively). Moreover, for most patients, category cNX (suggesting regional lymph nodes cannot be assessed) is considered invalid and should be listed as cN0, unless the patient has been previously subjected to axillary dissection.

Assessment of the metastases (M) stage involves categorizing patients into M0 or M1 using clinical examination. The M0 category indicates no clinical or radiographic evidence of distant metastases; however, this stage is designated cM0 since category $\mathrm{pM} 0$ is invalid (4). The designation pM1 may be used for patients with histologically proven metastases with at least 1 tumor deposit $>0.2 \mathrm{~mm}$. We also have cM0 (+), which indicates no clinical or radiographic evidence of distant metastases in the presence of histologically detected tumor deposits that are $<2 \mathrm{~mm}$ in circulating blood, bone marrow, or other non-regional nodal tissues, without symptoms of metastases. The designation M1 indicates distant metastases, where cM1 is detected clinically or radiographically and pM1 is histologically detected with at least 1 tumor deposit $>0.2 \mathrm{~mm}$. Finally, if a patient is assigned the M1 category, he/she is categorized as stage IV of the disease. The patient remains in stage IV regardless of response to any preoperative systemic therapy; however, if the patient has not received preoperative therapy, the stage should be updated if postoperative imaging within 4 months of diagnosis reveals distant metastases.

\section{Prognostic staging}

Reflected in the $8^{\text {th }}$ edition of the AJCC staging system, prognostic staging incorporates tumor grade, hormone receptors and oncogene status [estrogen receptors (ER), progesterone receptors (PR), and human epidermal growth factor receptor 2 (HER2)], and multigene panel results, in addition to the anatomic staging system discussed above.

\section{Tumor grade}

The AJCC $8^{\text {th }}$ edition manual highlights that, along with other factors, such as proliferative index, hormone receptor expression, and gene expression profiles, grade is a key assessment of tumor differentiation, which in turn is an important tool for prognosis. Tumor grade used by the manual for staging is defined by the histologic grading system of Scarff, Bloom, and Richardson and standardized by the Nottingham group (4). Regardless of hormonal therapy or chemotherapy, highgrade or poorly differentiated tumors have a worse prognosis than low-grade or well-differentiated tumors. Furthermore, the Survey, Epidemiology, and End Results Program of the National Cancer Institute revealed that histologic grade is a valuable prognostic factor, regardless of tumor size or number of positive lymph nodes (5).

\section{Hormone receptors and HER2}

According to the AJCC $8^{\text {th }}$ edition manual, hormone receptor and HER2 status need to be determined for all invasive breast cancers. Previous studies have demonstrated that ER- and PR-positive tumors can be effectively treated with selective ER modulators (SERMs), such as tamoxifen, to slow or stop tumor progression. Moreover, the higher the hormone receptor expression, the more effective the treatment becomes $(6,7)$. While ER- and PR-positive tumors are most likely to respond to SERMs, the response rate is lower for ER-positive and PR-negative, ER-negative and PR-positive, and ER-negative and PRnegative tumors, in descending order (6-8). 
Gene amplification or protein overexpression of the oncogene HER2 in untreated patients, whether node-positive or node-negative, has been associated with a worse prognosis $(9,10)$. Since HER2 positivity is associated with poor differentiation, it is more likely to be observed in high-grade invasive ductal carcinoma than invasive lobular carcinoma (11). Moreover, it is also associated with higher cell proliferation rates and hormone receptor negativity (12-14). The emergence of anti-HER2 targeted therapies has drastically improved the prognosis of patients with HER2-positive breast cancer. Particularly, the use of the monoclonal antibody trastuzumab in combination with a chemotherapeutic regimen significantly improves the disease-free and overall survival of these women (15).

\section{Biological subtypes}

In addition to tumor grade, hormone receptor, and oncogene expression, breast cancers vary widely on a genetic basis and this variation plays a significant role in prognosis. Four subtypes of breast cancer have been identified by genomic analysis: Luminal A, Luminal B, HER2-like, and Basal-like $(16,17)$. Categorizing cancers in such manner guides clinicians in both prognosis and treatment (18).

Particularly, luminal A-type tumors are deemed to have a favorable prognosis since they are typically low-grade invasive ductal carcinomas (not otherwise specified type) or special types, including tubular, cribiform, or mucinous carcinomas. This subtype is responsive to endocrine therapy but has a poor response to traditional chemotherapy. In contrast, luminal B-type tumors are typically poorly differentiated and respond better to traditional chemotherapy than endocrine therapy. Although HER2-like tumors previously had the worst prognosis among all subtypes, the introduction of anti-HER2 therapy has drastically improved prognosis of patients with these cancers. Finally, basal-like tumors, which usually have a triple-negative (ERnegative, PR-negative, and HER2-negative) phenotype, have the worst

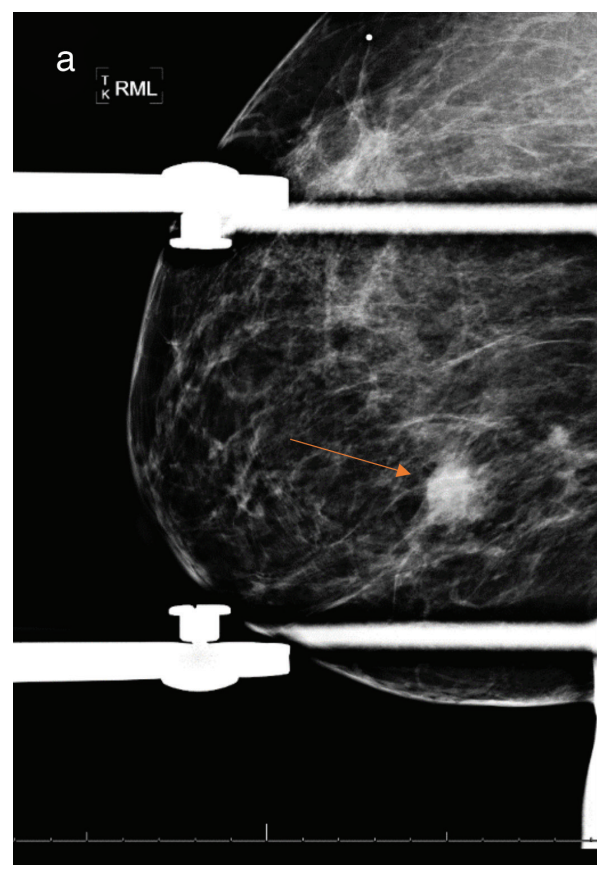

prognosis and are the most challenging to treat with adjuvant therapy $(4,16,17)$.

\section{Multigene panels}

Multigene panels can be used to obtain expression levels of multiple genes in breast cancer tissue. Many of these panels are useful prognostic tools; particularly, the Oncotype DX Breast Recurrence Score (Genomic Health, Redwood City, Calif), which measures 21 genes to predict likelihood of recurrence, has been incorporated into the updated staging system $(19,20)$. Despite this, one disadvantage of multigene panels is the substantial cost currently associated with their use. Thus, the AJCC $8^{\text {th }}$ edition manual states that hormone receptor and HER2 expression should be tested before obtaining a multigene panel and that the panel should be used only for certain subsets of cancers. Specifically, smaller (T1-T2) node-negative, hormone receptor-positive, HER2-negative tumors, and multigene panels may be incorporated into prognostic staging. When these tumors have an Oncotype DX score of $<11$, they can be considered stage IA, which can result in a downstage (4).

\section{Implementing the new staging system and challenges}

Upon the implementation of the AJCC $8^{\text {th }}$ edition staging system, many patients are restaged to better reflect their prognosis (Figures 1-3). A previous analysis was conducted to compare the $7^{\text {th }}$ edition to the revised $8^{\text {th }}$ edition staging system using the data obtained from 501,451 women in the National Cancer Database diagnosed from 2004 to 2014, excluding patients who underwent neoadjuvant chemotherapy. It was found that $23 \%$ of patients in stages I-III were downstaged and $19 \%$ of patients in stages I-III were upstaged (3).

Several studies involving large cohorts have revealed that these changes to patients' stages reflect more refined stratification and prediction of
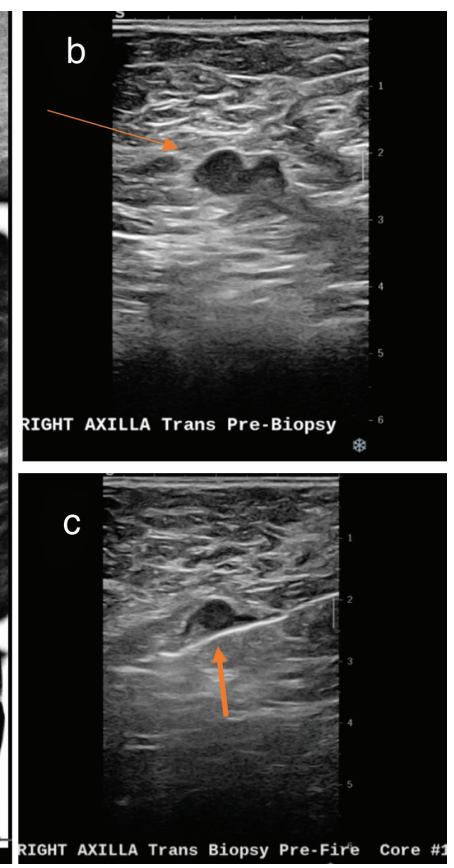

Figure 1. A 34-year-old woman with right breast cancer (a), $2.5 \mathrm{~cm}$, with a single metastatic axillary adenopathy identified with axillary ultrasound (b), and needle biopsy (c). Anatomic T2N1M0, Stage IIA (AJCC $7^{\text {th }}$ edition).

Patient's pathologic prognostic factors: Grade 2, ER, PR, and HER2-negative, which places her at stage IIIB (AJCC $8^{\text {th }}$ edition) 
disease outcome. One study including 3327 patients who underwent surgery as an initial intervention at the University of Texas MD Anderson Cancer Center database revealed that the incorporation of grade, ER, and HER2 status into AJCC prognostic staging provided a more refined stratification in terms of patients' disease-specific survival (21). Furthermore, an analysis of 54,727 patients in the California Cancer Registry revealed that the AJCC $8^{\text {th }}$ edition prognostic stage provided more accurate prognostic information than the anatomic stage alone (22).

Despite the benefits associated with incorporating prognostic markers into breast cancer staging, there are challenges to the implementation of this new staging system. In many parts of the world, biological markers and multigene panels are not routinely available (23). Also,

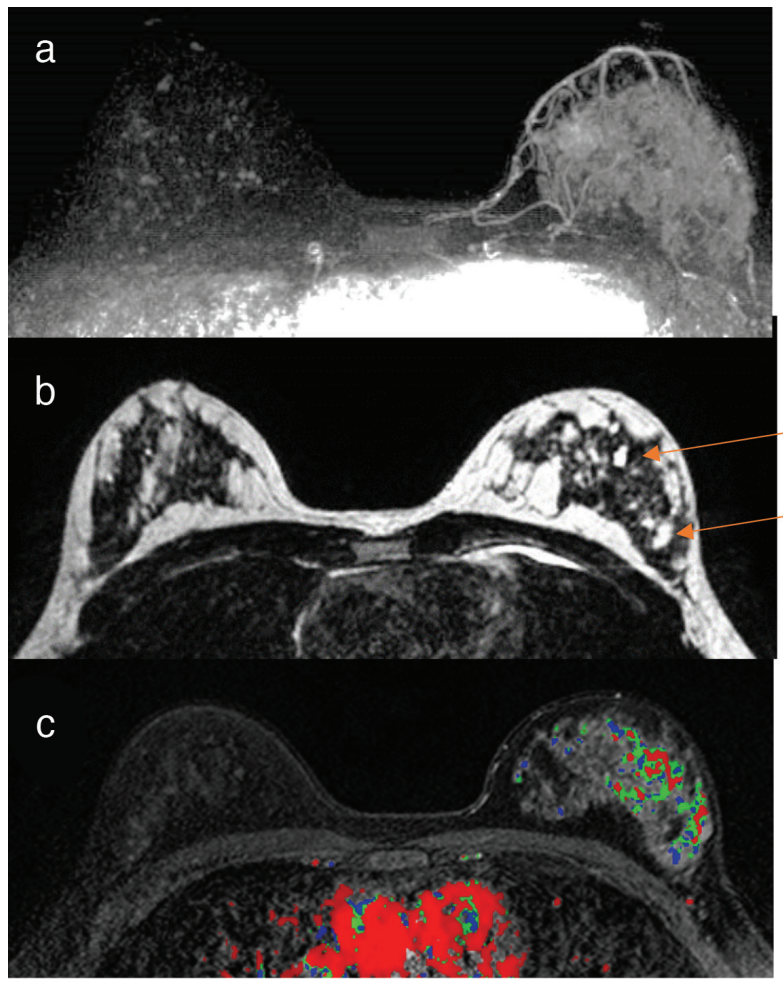

Figure 2. A 47-year-old pre-menopausal woman with a strong family history of breast cancer undergoing annual MRI screening. Figure reveals axial dynamic contrast-enhanced (DCE) MRI findings. (a) Maximum intensity projection (MIP) reveals cancer occupying a substantial portion of her left breast, (b) T2-weighted imaging reveals associated cysts (arrows) consistent with the micropapillary component, and (c) dynamic early subtraction image with overlying time intensity map. There is diffuse, non-mass-like enhancement involving all four quadrants of the left breast with no evidence of nipple involvement or axillary lymphadenopathy identified in the remainder of the MRI study.

Mastectomy pathology revealed multifocal invasive ductal carcinoma, grade 1, largest tumor measuring $56.0 \times 25.0 \mathrm{~mm}$. Additional foci of invasive carcinomas ranged in size from $1.0 \mathrm{~mm}$ to $7.0 \mathrm{~mm}$. Extensive ductal carcinoma in situ, Nuclear Grade 1, micropapillary and papillary types, measuring $95.0 \times 75.0 \mathrm{~mm}$. No axillary nodes were involved in pathology.

Anatomic T3NOMO, Stage IIB (AJCC $7^{\text {th }}$ edition).

Patient's pathologic prognostic factors: Grade 1, ER, PR, and HER2positive, which places her at stage IB (AJCC $8^{\text {th }}$ edition).

AJCC: American Joint Committee on Cancer; MRI: Magnetic resonance imaging; ER: Estrogen receptors; PR: Progesterone receptors; HER2: Human epidermal growth factor receptor 2 even in other parts of the world where biomarkers are more accessible, such as Europe, barriers in policy, reimbursement, and regulation have also delayed the widespread adoption of prognostic testing compared to the US (24). Therefore, the continued use of anatomic TNM staging in these regions emphasizes both its relevance and consistent usage.

\section{Conclusion}

The $8^{\text {th }}$ edition of the AJCC Cancer Staging Manual incorporates validated prognostic molecular biomarkers with standard tumor $(\mathrm{T})$, node $(\mathrm{N})$, and metastasis $(\mathrm{M})$ anatomic categories. Given the large number of possible combinations of T, $\mathrm{N}$, and $\mathrm{M}$ categories combined with grade, ER, PR, and HER2 status, integrating prognostic staging into multidisciplinary breast cancer care will be more complicated than with anatomic staging. Despite these challenges, prognostic staging facilitates more refined and accurate stratification of patients regarding

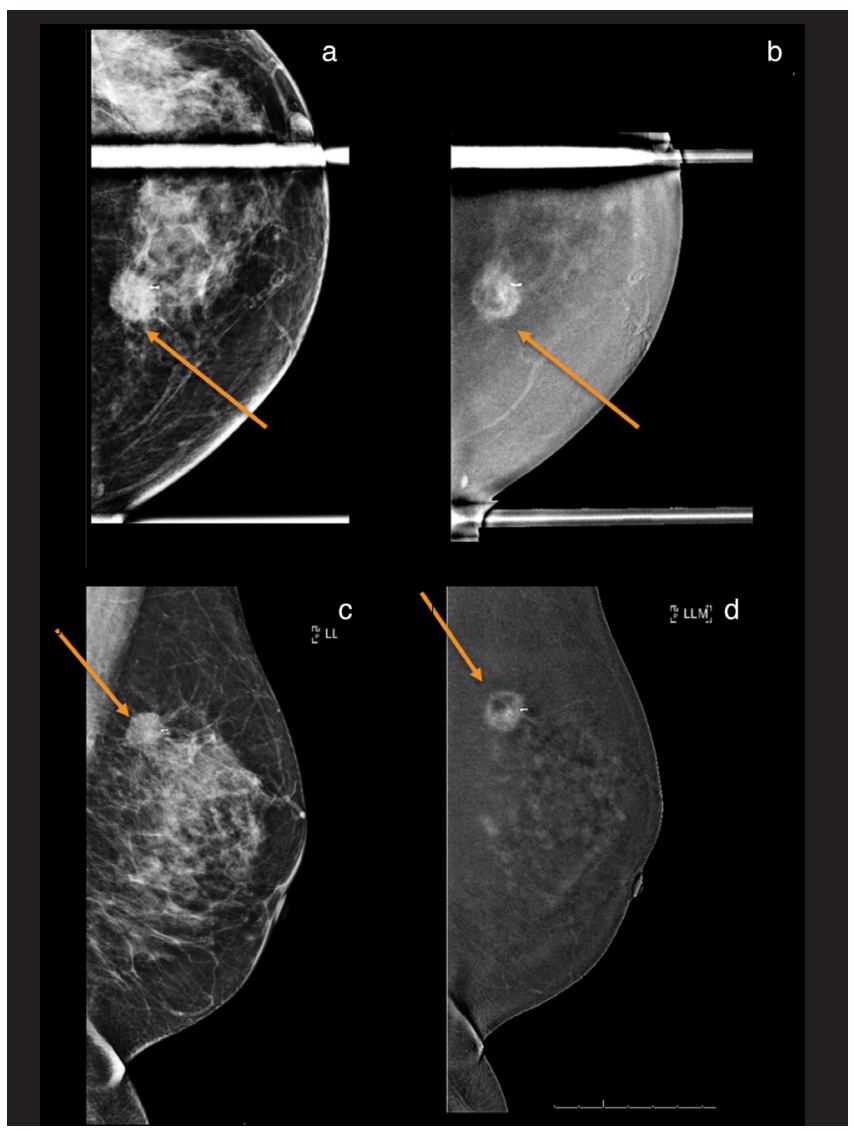

Figure 3. A 49-year-old woman with left breast invasive ductal cancer, grade 3.

Dual energy contrast-enhanced mammogram was performed to assess disease extent using iodinated low osmolar contrast injected at $3 \mathrm{ml} / \mathrm{sec}$. Spot compression craniocaudal low energy (a), high energy (b), left lateromedial low (c), and high energy (d) images reveal a $1.7 \mathrm{~cm}$ round mass with speculated margins. There was no palpable or imaging-detected lymphadenopathy in the axillary or internal mammary chains. Immunohistochemistry revealed ER/PRnegative cancer, HER2-negative cancer, and 90\% Ki-67.

Anatomic T1N0M0, Stage IA (AJCC $7^{\text {th }}$ edition)

Patient's pathologic prognostic factors: Grade 3, ER-, PR-, and HER2negative, which places her at Stage IIA (AJCC $8^{\text {th }}$ edition). The patient went on to receive neoadjuvant chemotherapy as first-line treatment.

AJCC: American Joint Committee on Cancer; ER: Estrogen receptors; PR: Progesterone receptors; HER2: Human epidermal growth factor receptor 2 
survival outcomes than anatomic staging alone, thereby ultimately allowing clinicians to better serve their patients. Although prognostic staging is preferred for patient care, anatomic staging is retained as a key component of cancer care in regions of the world where biomarker tests are not routinely available (4).

Peer-review: Externally peer-reviewed.

\section{Authorship Contributions}

Conception: H.Z., B.E.D.; Design: H.Z., B.E.D.; Supervision: H.Z., B.E.D.; Data Collection and/or Processing: H.Z., B.E.D.; Analysis and/or Interpretation: H.Z., B.E.D.;Literature Search: H.Z., B.E.D.; Writing: H.Z., B.E.D.; Critical Review: H.Z., B.E.D.

Conflict of Interest: No conflict of interest was declared by the authors.

Financial Disclosure: The authors declared that this study received no financial support.

\section{References}

1. Breastcancer.org. U.S. Breast Cancer Statistics. 2021. Last Accessed Date: 28.02.2021. Available from: https://www.breastcancer.org/symptoms/ understand_bc/statistics [Crossref]

2. Amin MB, Greene FL, Edge SB, Compton CC, Gershenwald JE, Brookland RK, et al. The Eighth Edition AJCC Cancer Staging Manual: continuing to build a bridge from a population-based to a more "personalized" approach to cancer staging. CA Cancer J Clin 2017; 67: 93-99. (PMID: 28094848) [Crossref]

3. Plichta JK, Campbell BM, Mittendorf EA, Hwang ES. Anatomy and breast cancer staging: is it still relevant? Surg Oncol Clin N Am 2018; 27: 51-67. (PMID: 29132565) [Crossref]

4. Hortobagyi GN, Connolly JL, D’Orsi CJ, Edge SB, Mittendorf EA, et al. Breast. In: Amin MB, Edge S, Greene F, et al, eds; American Joint Committee on Cancer. AJCC cancer staging manual. 8th ed. New York, NY: Springer, 2017.

5. Schwartz AM, Henson DE, Chen D, Rajamarthandan S. Histologic grade remains a prognostic factor for breast cancer regardless of the number of positive lymph nodes and tumor size: a study of 161708 cases of breast cancer from the SEER Program. Arch Pathol Lab Med 2014; 138: 10481052. (PMID: 25076293) [Crossref]

6. Davies C, Godwin J, Gray R, Clarke M, Cutter D, Darby S, et al. Early Breast Cancer Trialists' Collaborative Group (EBCTCG). Relevance of breast cancer hormone receptors and other factors to the efficacy of adjuvant tamoxifen: patient-level meta-analysis of randomised trials. Lancet 2011; 378: 771-784. (PMID: 21802721) [Crossref]

7. Barnes DM, Harris WH, Smith P, Millis RR, Rubens RD. Immunohistochemical determination of oestrogen receptor: comparison of different methods of assessment of staining and correlation with clinical outcome of breast cancer patients. Br J Cancer 1996; 74: 14451451. (PMID: 8912543) [Crossref]

8. Hammond ME, Hayes DF, Dowsett M, Allred DC, Hagerty KL, Badve S; American Society of Clinical Oncology; College of American Pathologists. American Society of Clinical Oncology/College of American Pathologists guideline recommendations for immunohistochemical testing of estrogen and progesterone receptors in breast cancer (unabridged version). Arch Pathol Lab Med 2010; 134: e48-e72. doi: 10.5858/134.7.e48. (PMID: 20586616) [Crossref]

9. Slamon DJ, Clark GM, Wong SG, Levin WJ, Ullrich A, McGuire WL. Human breast cancer: correlation of relapse and survival with amplification of the HER-2/neu oncogene. Science 1987; 235: 177-182. (PMID: 3798106) [Crossref]
10. Ross JS, Slodkowska EA, Symmans WF, Pusztai L, Ravdin PM, Hortobagyi GN. The HER-2 receptor and breast cancer: ten years of targeted anti-HER-2 therapy and personalized medicine. Oncologist 2009; 14: 320-368. (PMID: 19346299) [Crossref]

11. Rosenthal SI, Depowski PL, Sheehan CE, Ross JS. Comparison of HER-2/neu oncogene amplification detected by fluorescence in situ hybridization in lobular and ductal breast cancer. Appl Immunohistochem Mol Morphol 2002; 10: 40-46. (PMID: 11893034) [Crossref]

12. Eccles SA. The role of c-erbB-2/HER2/neu in breast cancer progression and metastasis. J Mammary Gland Biol Neoplasia 2001; 6: 393-406. doi: (PMID: 12013529) [Crossref]

13. Piccart $M$, Lohrisch $C$, Di Leo A, Larsimont $D$. The predictive value of HER2 in breast cancer. Oncology 2001;61(Suppl 2):73-82. (PMID: 11694791) [Crossref]

14. Yarden Y. Biology of HER 2 and its importance in breast cancer. Oncology 2001;61(Suppl 2):1-13. (PMID: 11694782) [Crossref]

15. Slamon D, Eiermann W, Robert N, Pienkowski T, Martin M, Press M, et al; Breast Cancer International Research Group. Adjuvant trastuzumab in HER2-positive breast cancer. N Engl J Med 2011; 365: 1273-1283. (PMID: 21991949) [Crossref]

16. Konecny G, Pauletti G, Pegram M, Untch M, Dandekar S, Aguilar Z, et al. Quantitative association between HER-2/neu and steroid hormone receptors in hormone receptor-positive primary breast cancer. J Natl Cancer Inst 2003; 95: 142-153. (PMID: 12529347) [Crossref]

17. Eiermann W, Rezai M, Kümmel S, Kühn T, Warm M, Friedrichs K, et al. The 21-gene recurrence score assay impacts adjuvant therapy recommendations for ER-positive, node-negative and node-positive early breast cancer resulting in a risk-adapted change in chemotherapy use. Ann Oncol 2013; 24: 618-624. (PMID: 23136233) [Crossref]

18. Coates AS, Winer EP, Goldhirsch A, Gelber RD, Gnant M, PiccartGebhart M, et al; Panel Members. Tailoring therapies--improving the management of early breast cancer: St Gallen International Expert Consensus on the Primary Therapy of Early Breast Cancer 2015. Ann Oncol 2015; 26: 1533-1546. (PMID: 25939896) [Crossref]

19. Xin L, Liu YH, Martin TA, Jiang WG. The era of multigene panels comes? The clinical utility of oncotype $\mathrm{dx}$ and mammaPrint. World J Oncol 2017; 8: 34-40. (PMID: 29147432) [Crossref]

20. Goncalves R, Bose R. Using multigene tests to select treatment for earlystage breast cancer. J Natl Compr Canc Netw 2013; 11: 174-182; quiz 182. (PMID: 23411384) [Crossref]

21. Mittendorf EA, Chavez-MacGregor M, Vila J, Yi M, Lichtensztajn DY, Clarke CA, et al. Bioscore: a staging system for breast cancer patients that reflects the prognostic significance of underlying tumor biology. Ann Surg Oncol 2017; 24: 3502-3509. (PMID: 28726077) [Crossref]

22. Weiss A, Chavez-MacGregor M, Lichtensztajn DY, Yi M, Tadros A, Hortobagyi GN, et al. Validation study of the American Joint Committee on cancer eighth edition prognostic stage compared with the anatomic stage in breast cancer. JAMA Oncol 2018; 4: 203-209. (PMID: 29222540) [Crossref]

23. Giuliano AE, Connolly JL, Edge SB, Mittendorf EA, Rugo HS, Solin LJ, et al. Breast cancer-major changes in the American Joint Committee on Cancer eighth edition cancer staging manual. CA Cancer J Clin 2017; 67: 290-303. Erratum in: CA Cancer J Clin 2017; 67: 345. (PMID: 28294295) [Crossref]

24. Horgan D, Ciliberto G, Conte P, Baldwin D, Seijo L, Montuenga LM, et al. Bringing greater accuracy to europe's healthcare systems: the unexploited potential of biomarker testing in oncology. Biomed Hub 2020; 5: 182-223. (PMID: 33564664) [Crossref] 